\title{
Let's talk about sex history: impact of a communication curriculum on medical student performance and perceived competency
}

\author{
Colleen K. Stockdale MD, MS, ${ }^{1}$ John Kippes MA, ${ }^{2}$ Marcy Rosenbaum PhD, ${ }^{3}$ \\ Marygrace Elson MD, $\mathrm{MME}^{1}$
}

Keywords: Sexual history, communication, curriculum, medical student, education

\begin{abstract} Introduction: Communication in general, but especially regarding difficult topics such as sexual health is inadequately addressed in undergraduate medical education. Students often feel they are poorly trained in this area due to a lack of opportunities to practice taking sexual history. Thus, a communication curriculum was implemented to address this gap, including formal small group practice sessions on taking a sexual history during the Obstetrics and Gynecology (OB/GYN) clerkship.

Aim: Determine whether medical students who completed a sexual history communication curriculum performed better during a formal standardized patient based assessment (PBA) at the conclusion of the OB/GYN clerkship than historical control medical students, and assess medical student perception about taking a sexual history.

Methods: Retrospective study comparing 3 specific PBA standardized patient rating questions and 5 questions gathering medical student feedback at a single academic institution. All $(n=71)$ medical students between January and June 2011 were queried at the
\end{abstract}

conclusion of their OB/GYN clerkship prior to implementation of the sexual history curriculum in July 2011 with responses compared to all subsequent $(n=432)$ medical students through June 2014.

Main Outcome Measures: Cochran-MantelHaenszel was used to compare scores by clerkship year and block with $p<0.05$ significant.

Results: There was no change in medical student PBA performance or medical student perception regarding ability to take a sexual history following implementation of the sexual history communication curriculum. However, in response to "what other instruction or experiences could the clerkship provide to help you gain competency," the theme was more practical experience.

Conclusion: Learners require different formats to promote and enhance learning which may not have been measured with the outcomes chosen for this study. This may be best achieved by implementation of a formal curriculum including explicit supplementation to incorporate additional experience with sexual history taking.

Please cite this paper as: Stockdale CK, Kippes J, Rosenbaum M, Elson M. Let's talk about sex history: impact of a communication curriculum on medical student performance and perceived competency. Proc Obstet Gynecol. 2018;8(3):Article 2 [ 10 p.]. Available from: http://ir.uiowa.edu/pog/. Free full text article.

Corresponding author: Colleen Stockdale, MD, MS, Department of Obstetrics and Gynecology, 200 Hawkins Drive, Iowa City, IA 52246, Phone: 319-384-6849, Fax: 319-384-8620. Email: colleen-stockdale@uiowa.edu

Financial Disclosure: The authors report no conflict of interest.

Copyright: (c) 2018 Stockdale et al. This is an open-access article distributed under the terms of the Creative Commons Attribution License, which permits unrestricted use, distribution, and reproduction in any medium, provided the original author and source are credited. 
${ }^{1}$ Department of Obstetrics and Gynecology, University of lowa Carver College of Medicine, lowa City, IA

${ }^{2}$ Department of Student Life, Dakota Wesleyan University, Mitchell, SD

${ }^{3}$ Department of Family Medicine, University of lowa Carver College of Medicine, lowa City, IA

\section{Introduction}

Despite the importance of communication between physician and patient, communication in general is inadequately addressed in medical education. While significant time is devoted to this in pre-clinical training in some institutions, continuing and reinforcing this training while medical students are in clinical clerkships has often been inadequate. ${ }^{1}$ Furthermore, difficult topics in communication, e.g. "taking a sexual history" are often neglected altogether. ${ }^{2,3}$ Thus, taking a sexual history is a difficult and often uncomfortable experience for medical students. ${ }^{4}$ This translates into clinicians who feel ill prepared to address sexual health concerns and thus seldom initiate discussion. ${ }^{2,5}$

In addition to deficiencies in the medical curriculum regarding communication in general, poor teaching strategies have contributed to ineffective learning especially for difficult topics such as sexual history. ${ }^{6}$ Data support the provision of opportunities to learn and practice interviewing and to receive feedback on skills. ${ }^{1,2}$ Additionally, medical students often feel they are poorly trained in this area due to a lack of opportunities to practice taking sexual histories. ${ }^{6}$ Thus, a sexual history-taking communication curriculum during the Obstetrics and Gynecology (OB/GYN) clerkship at our institution was implemented to address this general gap in medical student education.

Prior to implementing the sexual history taking communication curriculum during the OB/GYN clerkship, medical students received at least a lecture concerning sexual history taking including advanced communication skills, terminology, patient and provider biases and assumptions in the first pre-clinical semester and were offered additional information during a pre-clinical "sex week" during the third pre-clinical semester which offered a broad overview of sexuality and sexual health across the lifespan with presentations and discussions by various professions and lay persons. However, medical students had little opportunity to practice. Subsequently medical students had little opportunity to practice with real patients during clerkships.

\section{Aims}

The purpose of this study was to 1) determine whether medical students who completed a sexual history-taking communication curriculum performed better during a formal standardized patient based assessment (PBA) at the conclusion of the OB/GYN clerkship than historical control medical students, and 2) assess medical students perceptions about taking a sexual history before and after implementation of the sexual history taking communication curriculum.

\section{Methods}

This is a retrospective case-control study to evaluate a sexual history-taking curriculum implemented during the 6 week OB/GYN clerkship academic year beginning 2011-2012 at a single 
academic institution.

The primary aim of the sexual history taking session is to provide an opportunity for students to have a safe, observed opportunity to practice taking a sexual history. Core skills of gathering information and building the relationship are reviewed, as well as exploring the need for sensitive and specific communication skills (e.g. open vs closed ended questions). The session provides medical students the opportunity to practice core and advanced communication skills required for sensitive topics with standardized patients in a safe environment.

This study was approved by the University of lowa, Carver College of Medicine Education Umbrella IRB Review Committee (retrospective review of sexual history curriculum intervention).

\section{Intervention}

The sexual history taking component was added to provide medical students an opportunity to practice taking a sexual history with two standardized patients in a safe environment. The sexual history-taking session, which was adapted from a similar session used at the University of Cambridge UK (https://www.each.eu/teaching/resource s/sexual-history-taking-genitourinarycambridge-110921/ ) involves a small group, role-play activity including two facilitators (who review components of sexual history and communication skills), two standardized patients, and four to six medical students. ${ }^{7}$ The session uses an Agenda-Led Outcomes Based Analysis (ALOBA) approach in which students identify what skills they want to work on and try out, complete a portion of the interview with a standardized patient, receive feedback from the standardized patient, peers and facilitators, and then have a chance to retry a brief part of the encounter based on the feedback they received. ${ }^{7}$ The second standardized patient provides a second (more challenging) opportunity for students to practice sexual history taking with similar group feedback and opportunity for second practice. It has been shown that interactive approaches to teaching sexual history taking are highly acceptable to participants and allows learners to reflect on and practice skills being taught. ${ }^{8}$

The patient scenarios include a young sexually active woman with vaginal discharge, probably sexually acquired (one regular partner, one indiscretion); a straightforward case of sexually transmitted infection. The second case involves a 70 year old woman, with vulvar irritation, not sexually acquired who is embarrassed and requires significant relationship building to discuss that she is not now sexually active because it is too sore and her concern that her husband may have had sex outside of their marriage.

\section{Assessment}

Participants: Cases include all medical students who completed the OB/GYN clerkship beginning July 2011 through December 2014 (seven semesters) following the implementation of the sexual history-taking communication curriculum. Controls include all medical students who completed the OB/GYN clerkship from January 1, 2011 through June 2011 (one semester) prior to implementation of the sexual history- 
taking communication curriculum. The $\mathrm{OB} / \mathrm{GYN}$ clerkship is a required (core) clerkship typically taken during the third year of medical school. A few medical students, primarily those enrolled in dual degree programs (master and dual doctorate degree) complete the OB/GYN clerkship following completion of their dual degree; later year of their medical school training. Given the small number of dual degree students involved, and unidentified individual responses we were not able to separate or remove those dual degree students for the analyses.

Data collection methods: Medical students were assessed at the conclusion of the OB/GYN clerkship at the time of their required Patient Based Assessment (PBA). The PBA consisted of three fifteen-minute problem-focused interactions with standardized patients who are trained to assess students regarding the scenario. One of these three cases required a sexual history, in addition to through history of present illness and past medical history for a patient with a chief complaint of vaginal discharge.

To evaluate medical student performance regarding sexual history taking, 3 questions were added to the vaginal discharge PBA patient scenario to assess best practices in sexual history taking (use of a transition statement, being non-judgmental, and use of direct questions), with responses on a 5 point scale from "strongly disagree" (1) to "strongly agree" (5):

1. Student provided a transition statement before asking my sexual history.

2. Student showed openness to sexual orientation and / or forms of sexual expression.

3. Student obtained sexual history in a clinical manner using direct questions.

Similarly, five questions were added to the medical student PBA evaluation to assess whether medical students felt the clerkship prepared them to take a sexual history with student responses on the same five point scale above for questions 1-3, and a four point scale from "not competent" (1) to "extremely competent" (4) for questions 4 and 5 :

1. On this rotation, I have been involved in the care of at least 1 patient with this chief complaint.

2. The OB/Gyn clerkship well prepared me for taking a sexual history from real patients.

3. The sexual history taking skills I gained on this clerkship will also help me take a sexual history from a male patient.

4. Please rate yourself in terms of your ability to take a sexual history prior to this clerkship.

5. As a result of this clerkship, how would you now rate yourself in terms of ability to take a sexual history?

Additionally, medical students were asked an open ended question: "What other instruction or experiences could the clerkship provide to help you gain competency in taking a sexual history from real patients?"

Thus, all medical students were evaluated by the standardized patient following the PBA vaginal discharge scenario involving sexual history-taking 
(three questions). Based on the number of standardized patient responses each block, we were able to determine that all students completed the student PBA evaluation (including five questions regarding sexual history-taking).

\section{Outcome Measures}

The data were analyzed using CochranMantel-Haenszel row mean, with Chisquare computed for each test. Responses were treated as values, with "strongly disagree" being lowest (1) and "strongly agree" being highest (5) for the three questions added for the standardized patient to evaluate. Responses for the five questions added for student self-evaluation were similarly treated as values, with "strongly disagree" (1) and "strongly agree" (5) for questions 1-3, and "not competent" being lowest (1) and "extremely competent" being highest (4) for questions 4 and 5 . A p-value less than 0.05 was considered significant.

Responses were grouped as blocks corresponding to each OB/GYN clerkship rotation and by year. Each academic year began in July and ended in June, including four blocks or OB/GYN clerkship rotations each semester for a total of eight blocks each year. Data were analyzed by block and by year for comparisons.

\section{Results}

All medical students completed the OB/GYN PBA including the patient with complaint of vaginal discharge at the conclusion of their six week OB/GYN clerkship and completed a PBA evaluation including the five additional questions to assess student selfcompetency regarding sexual historytaking. Thus, there were 71 historical controls and 432 responses following implementation of the sexual historytaking curriculum for comparison. The demographic characteristics by academic year (for third year OB/GYN clerkship) based on admissions information is presented in table 1.

Table 1. University of lowa Carver College of Medicine Admissions Demographic Information (for third year medical students by academic year)

\begin{tabular}{|c|c|c|c|c|c|c|c|c|}
\hline \multirow[b]{3}{*}{ Demographics } & \multicolumn{2}{|c|}{ 2010-2011 } & \multicolumn{2}{|c|}{ 2011-2012 } & \multicolumn{2}{|c|}{ 2012-2013 } & \multicolumn{2}{|c|}{ 2013-2014 } \\
\hline & \multicolumn{2}{|c|}{ (pre-intervention) } & \multicolumn{6}{|c|}{ (post-intervention) } \\
\hline & Age & Range & Age & Range & Age & Range & Age & Range \\
\hline \multirow[t]{2}{*}{ Mean age (years) } & 27 & $23-46$ & 26 & $24-37$ & 27 & $23-39$ & 27 & $23-55$ \\
\hline & $\mathrm{N}$ & $\%$ & $\mathrm{~N}$ & $\%$ & $\mathrm{~N}$ & $\%$ & $\mathrm{~N}$ & $\%$ \\
\hline Female & 68 & $49.6 \%$ & 59 & $47.2 \%$ & 64 & $45.1 \%$ & 59 & $41.0 \%$ \\
\hline Male & 69 & $50.4 \%$ & 66 & $52.8 \%$ & 78 & $54.9 \%$ & 85 & $59.0 \%$ \\
\hline White & 103 & $75.2 \%$ & 99 & $79.2 \%$ & 107 & $75.4 \%$ & 107 & $74.3 \%$ \\
\hline Asian & 19 & $13.9 \%$ & 8 & $6.4 \%$ & 12 & $8.5 \%$ & 24 & $16.7 \%$ \\
\hline African-American & 7 & $5.1 \%$ & 5 & $4.0 \%$ & 9 & $6.3 \%$ & 3 & $2.1 \%$ \\
\hline Other & 8 & $5.8 \%$ & 12 & $9.6 \%$ & 14 & $9.9 \%$ & 10 & $6.9 \%$ \\
\hline
\end{tabular}


The standardized patient with vaginal discharge completed feedback for each student including the three additional questions to assess sexual historytaking during the same time period for $100 \%$ response.

As demonstrated in Figure 1, there were no significant differences between the medical student PBA performances as assessed by the standardized patients. For question 1 (student provided a transition statement), the average score ranged from 3.47 to $3.59(p=0.66)$. For question 2 (student showed openness to sexual orientation and/or forms of sexual expression), the average score ranged from 3.85 to $3.94(p=0.78)$. For question 3 (student obtained sexual history in a clinical manner using direct questions), the average score ranged from 3.63 to $3.85(p=0.14)$. Interestingly, as depicted in Figure 1, scores varied for each question by year.

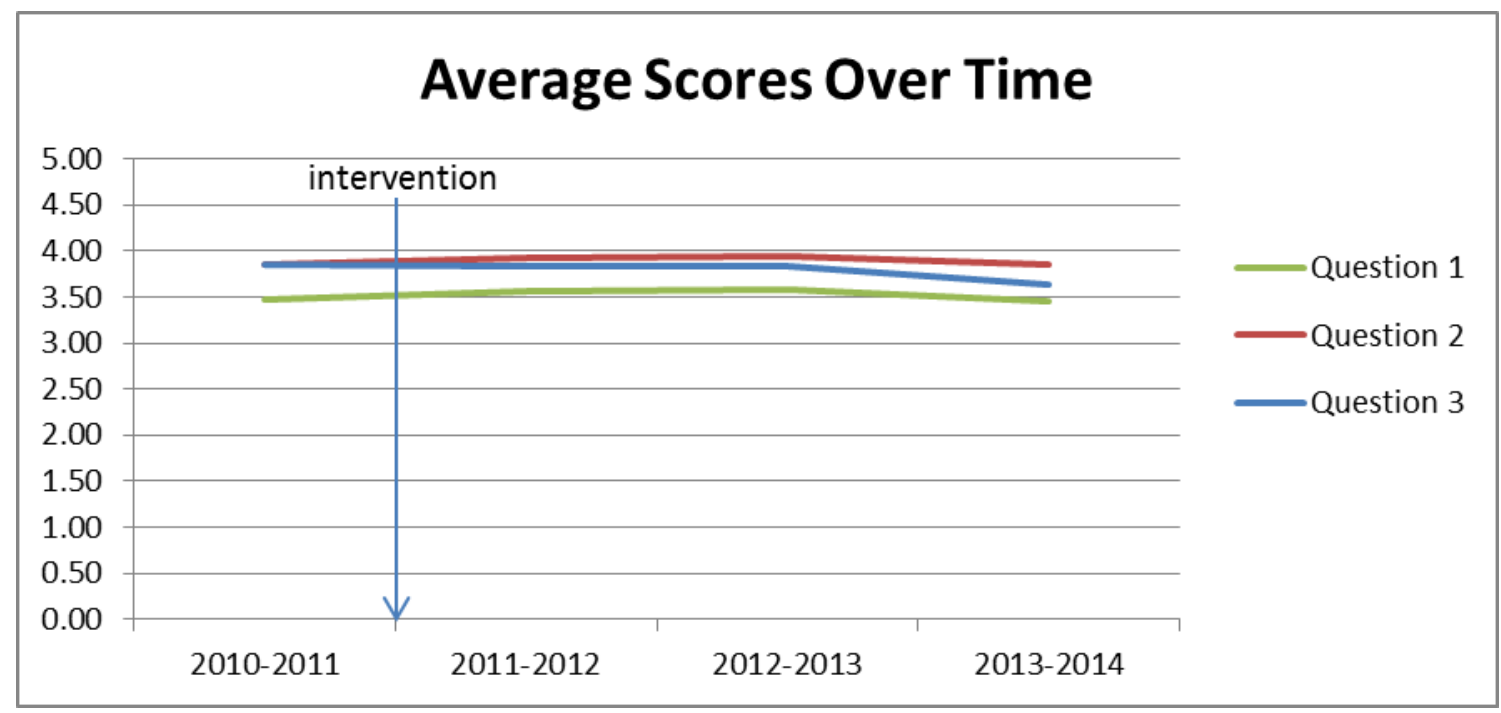

Standardized patient responses on a 5 point scale from "strongly disagree" (1) to "strongly agree" (5). Questions: 1. Student provided a transition statement before asking my sexual history.

2. Student showed openness to sexual orientation and /or forms of sexual expression.

3. Student obtained sexual history in a clinical manner using direct questions.

\section{Figure 1. Student performance on the patient based assessment}

As demonstrated in Figure 2, there were no significant differences regarding student perception regarding ability to take a sexual history immediately following implementation of the sexual history communication curriculum. For question 1 (student involved in the care of at least 1 patient with this chief complaint), the average score ranged from 4.08 to $4.34(p=0.28)$. For question 2 (clerkship prepared me for taking a sexual history from real patients), the 
average score ranged from 4.03 to 4.26 $(p=0.21)$. For question 3 (sexual history taking skills gained on the clerkship will help me take a sexual history from a male patient), the average score ranged from 3.82 to $4.07(p=0.14)$. For question 4 (self-rating of ability to take a sexual history prior to the clerkship - 4 point scale), the average score ranged from 2.34 to $2.56(p=0.18)$. For question 5 (how would you now rate yourself in terms of ability to take a sexual history 4 point scale), the average score ranged from 3.00 to $3.24(p=0.14)$. Again, it is interesting to note the scores varied for each question by year. While the difference between each medical student's score for their ability to take a sexual history following the OB/GYN clerkship compared to prior to the clerkship was significant (question 5 compared with question $4(p<0.001))$, there was no difference between the change in scores and year pre and postintervention $(p=0.25)$.

Although there was no change in PBA standardized patient or medical student response to specific questions following implementation of the sexual history communication curriculum, in response to "what other instruction or experiences could the clerkship provide to help you gain competency" the theme by medical students was more practical experience.

\section{Discussion}

We found no statistically significant difference in scores following implementation of a sexual historytaking communication curriculum regarding medical student performance or perception regarding ability to take a sexual history. Although the future practice of sexual medicine may be influenced by the effectiveness and implementation of further education in this area, it is not clear that the addition of a sexual history curriculum improved the clinical ability or self-perceived ability of medical students following their OB/GYN clerkship.

The significance of students' consistent responses of 'more practical experience' in answer to the question "what other instruction or experiences could the clerkship provide to help you gain competency" is difficult to interpret. The educational intervention was implemented specifically to provide students practical experience in sexual history taking, with an opportunity for feedback while on the OB/GYN clerkship. However, it provides experience with simulated rather than real patients. Thus, this response does not necessarily devalue the contribution of the educational activity to their learning, but instead may be viewed as not sufficient for achieving competency in these skills. Rather, medical students may perceive that repeated practice with real patients would contribute to their learning. This theme has also been identified by others, as repeat exposure allows students to become more comfortable and confident. ${ }^{6,9}$

We chose to use an objectives-oriented approach to determine the impact of the sexual history-taking curriculum. While this is a simple and well known measure, it has several disadvantages. It promotes tunnel vision, in that it focuses on predetermined objectives; objectives may be poorly chosen (infeasible), and educators may teach to the objectives. ${ }^{10}$ Case-in-point, the second and third PBA questions are vague and the response may vary by 
the standardized patient assessor despite extensive training for uniform assessment standards. Thus, the outcome measures (questions) used for this study may not have been adequately focused to measure the specific effect of the educational intervention.

\section{Average Scores Over Time}

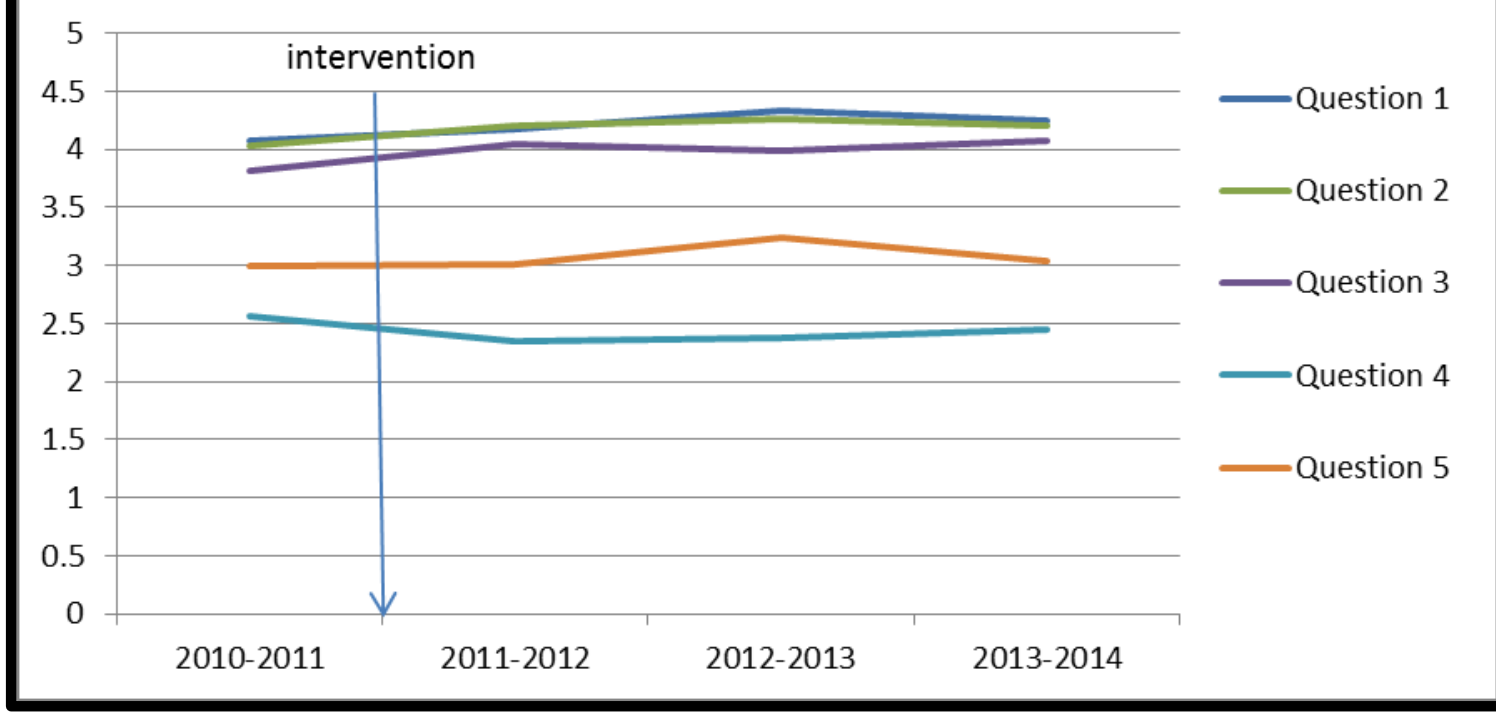

Student responses on a 5 point scale from "strongly disagree" (1) to "strongly agree" (5), questions 1-3, and a 4 point scale from "not competent" (1) to "extremely competent" (4), questions 4 and 5.

Questions:

1. On this rotation, I have been involved in the care of at least 1 patient with this chief complaint (5 point).

2. OB/Gyn clerkship well prepared me for taking a sexual history from real patients (5 point).

3. Sexual history taking skills I gained on this clerkship will also help me take a sexual history from a male patient (5 point).

4. Please rate yourself in terms of your ability to take a sexual history prior to this clerkship (4 point).

5. As a result of this clerkship, how would you now rate yourself in terms of ability to take a sexual history (4 point).

Figure 2. Medical student perception

While the student self-perception questions are specific to medical student's perceptions, we were unable to determine whether or not medical students valued or felt there was an impact from the sexual history-taking component specifically. Additionally, despite similar medical student responses before and after implementation of the sexual history 
Proceedings in Obstetrics and Gynecology, 2018;8(3):2

communication curriculum, we did find a significant difference in student perceptions of their competence in sexual history-taking prior to versus after the clerkship experience as a whole. To better assess whether or not the sexual history curriculum is perceived as beneficial to the medical students, questions specifically asking them to assess the added value of these sessions would be necessary.

Additionally given the limited research regarding sexual history-taking we were unable to identify validated instruments for our study, thus the questions were developed by experienced educators (MR, ME) prior to implementation of the sexual history curriculum. Though imperfect, the questions do reflect student perceptions regarding their sexual history taking skills. Moreover, student responses regarding their ability to take a sexual history suggest the need for additional education and practice.

While studies have found medical school training on sexual health issues including sexual history taking to be deficient, ${ }^{11}$ there remains a lack of consensus as to what should constitute a sexual health curriculum. A review from 2008 identified that about half of US medical schools have formal sexual heath curricula. ${ }^{11}$ Among programs with formal curricula, didactic training is most often used for teaching sexual historytaking. As with Coverdale, we agree curriculum design should follow standard methods including ongoing and thorough evaluations of the content and processes of implementation. ${ }^{2}$ Though evaluation is essential, evidence shows that communication skills can be learned, but such skills are not easily assessed by traditional methods. $^{12}$

\section{Conclusions}

We agree with our learners that communication in general, and sexual history-taking communication skills specifically, improve as we gain 'more practical experience'. Likely our outcome measures were unable to assess the 'practical experience' rather than a lack of effect of providing an opportunity for students to have a safe, observed practice of core and new skills necessary for taking the sexual history. Learners require different formats to promote and enhance learning which may not have been measured with the outcomes chosen for this study. Furthermore, PBA scores may not fully reflect changes in medical student performance as they do not necessarily capture the learning that occurs in sessions. Additionally, sessions alone without substantial practical experience may lead to less uptake and incorporation of learning. There needs to be sufficient pre-data for comparison with post-data. Other measures may be more appropriate including pre-session PBAs early in the clerkship compared to post-session PBAs, observation of students with real patients focusing on the specific skills emphasized in the sessions, and direct assessment of medical student perspectives on the value of these sessions. This may be best achieved by implementation of a formal curriculum including explicit supplementation to incorporate additional experience with sexual history taking.

Abstract presented at 2016 CREOG \& APGO Annual Meeting, March 2-5, 2016, New Orleans, 
$L A$

\section{References}

1. Rosenbaum ME. Dis-integration of communication in healthcare education: Workplace learning challenges and opportunities. Patient Educ Couns. 2017 Nov;100(11):2054-2061.

https://doi.org/10.1016/j.pec.2017.05.03

$\underline{5}$ Epub 2017 Jun 3. PubMed PMID: 28602566.

2. Coverdale $\mathrm{JH}$, Balon $\mathrm{R}$, Roberts $\mathrm{LW}$. Teaching sexual history-taking: a systematic review of educational programs. Acad Med. 2011 Dec;86(12):1590-5.

https://doi.org/10.1097/ACM.0b013e318 234ea41 PubMed PMID: 22030763.

3. Parish SJ, Clayton AH. Sexual medicine education: review and commentary. J Sex Med. 2007 Mar;4(2):259-67; quiz $268 . \quad$ https://doi.org/10.1111/i.17436109.2007.00430.x PubMed PMID: 17367420 .

4. Stanton M, Anderson D, Szerlip HM. Teaching the sexual history: a tutorial approach. Acad Med. 1997 May; $72(5): 430$.

https://doi.org/10.1097/00001888199705000-00059 PubMed PMID: 10676353.

5. Rosen R, Kountz D, Post-Zwicker T, Leiblum $S$, Wiegel $M$. Sexual communication skills in residency training: the Robert Wood Johnson model. J Sex Med. 2006 Jan;3(1):37-46. https://doi.org/10.1111/i.17436109.2005.00135.x PubMed PMID: 16409216.

6. Jayasuriya AN, Dennick R. Sexual history-taking: using educational interventions to overcome barriers to learning. Sex Education. 2011;11(1):99112. https://doi.org/10.1080/14681811.2011. $\underline{538155}$
7. Silverman JD, Kurtz SM, Draper J. Skills for communicating with patients. Oxford, UK: Radcliffe Medical Press; 1998. https://www.each.eu/teaching/resources Isexual-history-taking-genitourinarycambridge-110921/

8. Skelton JR, Matthews PM. Teaching sexual history taking to health care professionals in primary care. Med Educ. 2001 Jun;35(6):603-8. https://doi.org/10.1046/j.13652923.2001.00926.x PubMed PMID: 11380865.

9. Cushing A, Evans D, Hall A. Medical students' attitudes and behaviour towards sexual health interviewing: short- and long-term evaluation of designated workshops. Med Teach. 2005 Aug;27(5):422-8. https://doi.org/10.1080/0142159050004 6502 PubMed PMID: 16147795.

10. Cook DA. Twelve tips for evaluating educational programs. Med Teach. 2010;32(4):296-301. https://doi.org/10.3109/0142159090348 0121 PubMed PMID: 20353325.

11. Malhotra S, Khurshid A, Hendricks KA, Mann JR. Medical school sexual health curriculum and training in the United States. J Natl Med Assoc. 2008 Sep;100(9):1097-106. https://doi.org/10.1016/S00279684(15)31452-8 PubMed PMID: 18807442.

12. Shirazi M, Labaf A, Monjazebi F, Jalili M, Mirzazadeh M, Ponzer S, Masiello I. Assessing medical students' communication skills by the use of standardized patients: emphasizing standardized patients' quality assurance. Acad Psychiatry. 2014 Jun;38(3):354-60. https://doi.org/10.1007/s40596-0140066-2 Epub 2014 Apr 29. PubMed PMID: 24777713. 